\title{
Caracterização da normalidade do P300 em adultos jovens
}

\author{
Standard characterization of P300 in young adults
}

\author{
Cíntia Santos Silva Machadoㄹ, Angélika Caldeira Oliveira Carvalho², Paloma Luara Guerra e Silva ${ }^{3}$
}

RESUMO

\begin{abstract}
Objetivo: Comparar com a literatura os valores de latência e amplitude de P300 em adultos jovens brasileiros saudáveis a fim de estabelecer um padrão normativo para futuros estudos, e verificar se os valores encontrados na amostra possuem correlação com o sexo, situação proposta na metodologia do exame e fases do ciclo menstrual. Métodos: A amostra constou de 22 indivíduos saudáveis, sem queixa de desatenção, sem problemas neurológicos e auditivos, de 18 a 30 anos de idade. Todos os indivíduos foram submetidos à avaliação audiológica básica a fim de excluir aqueles com perda de audição. As respostas do P300 foram obtidas com equipamento AMPLAID MK22, de dois canais e em ambiente silencioso. Resultados: Os resultados de latência encontrados na amostra, depois de comparados à literatura, enquadraram-se melhor nos intervalos de latência entre 220 e 380 ms. Somente os valores de amplitude foram influenciados pelas variáveis sexo e fase do ciclo menstrual. Conclusão: Os valores de latência da amostra não sofreram influência da variável sexo, ciclo menstrual e situação, e aproximaram-se com mais fidedignidade aos valores de intervalos de latência entre 220 e 380 ms. Contudo, os intervalos para a amplitude sofreram influência das variáveis sexo e ciclo menstrual, mas não se enquadraram adequadamente aos valores de intervalo encontrados na literatura.
\end{abstract}

Descritores: Testes auditivos; Potenciais evocados auditivos; Potencial evocado P300; Adulto

\section{INTRODUÇÃO}

A audição é um dos cinco sentidos dos seres humanos, sendo a orelha um órgão muito sensível que nos possibilita perceber e interpretar ondas sonoras. O sentido auditivo é constituído por etapas, como a captação do som, percepção e por último, interpretação do mesmo. Essas etapas ocorrem devido às transformações de energia, iniciando pela sonora, passando pela mecânica, hidráulica e finalizando com a energia elétrica, que após atravessar o nervo coclear é transmitida aos centros auditivos do tronco encefálico e córtex cerebral. Existem exames que possibilitam a mensuração da função auditiva em todo seu percurso até o córtex cerebral, estando cada exame relacionado a uma ou mais porções da via auditiva ${ }^{(1-3)}$.

Para avaliar a audição no córtex auditivo pode-se utilizar o exame de potencial evocado auditivo de longa latência, classificado em potenciais exógenos e endógenos. Os exó-

Trabalho realizado na Pontifícia Universidade Católica de Minas Gerais PUC-Minas - Belo Horizonte (MG), Brasil.

(1) Mestre, Professora assistente do Curso de Fonoaudiologia da Pontifícia Universidade Católica de Minas Gerais - PUC-Minas e do Unicentro Izabela Hendrix - Belo Horizonte (MG), Brasil.

(2) Acadêmica do Curso de Fonoaudiologia da Pontifícia Universidade Católica de Minas Gerais - PUC-Minas - Belo Horizonte (MG), Brasil.

(3) Acadêmica do Curso de Fonoaudiologia da Pontifícia Universidade Católica de Minas Gerais - PUC-Minas - Belo Horizonte (MG), Brasil. Endereço para correspondência: Cíntia Santos Silva Machado. R. Cláudio Manuel, 521/2303, Bairro Funcionários, Belo Horizonte - MG, CEP 30140100. E-mail: cintias@ gmail.com

Recebido em: 6/3/2008; Aceito em: 17/8/2008 genos são influenciados principalmente pelas características físicas do estímulo (intensidade, freqüência), sendo os seus componentes $\mathrm{P}_{60}, \mathrm{~N}_{100}, \mathrm{P}_{160}(\mathrm{~N}$ corresponde a uma onda negativa e $\mathrm{P}$ corresponde a uma onda positiva, os valores que as acompanham, referem-se à latência em ms). Os endógenos são influenciados por eventos internos relacionados à função cognitiva (habilidades cognitivas, percepção ou atenção), sendo os seus componentes $\mathrm{N}_{200}$ e P300. Assim, o potencial evocado auditivo de longa latência endógeno, relacionado a um evento, que aparece com uma onda positiva aproximadamente aos 300ms após o estímulo é chamado de P300 ${ }^{(2-4)}$.

O P300 reflete principalmente a atividade do tálamo e córtex, estruturas que envolvem as funções de discriminação, integração e atenção do cérebro ao som e, por isso, é utilizado para detectar alterações neurais do processamento sequencial de informações, memória imediata e/ou tomada de decisões ${ }^{(2-7)}$.

Graficamente o P300 é caracterizado por uma onda de grande amplitude que surge aproximadamente a $300 \mathrm{~ms}$, gerada pela expectativa da percepção sonora de um estimulo chamado raro ${ }^{(5)}$, em contrapartida a outro estímulo chamado frequente; ele ainda pode ter um componente inicial $\left(\mathrm{P}_{3 \mathrm{a}}\right)$, notado quando o paciente ignora o estímulo e o componente tardio $\left(\mathrm{P}_{3 \mathrm{~b}}\right)$ quando há atenção. $\mathrm{O}$ subcomponente $\mathrm{P}_{3}$ será elucidado quando for maior a dificuldade do exame ${ }^{(8)}$.

Os trabalhos encontrados na literatura usam metodologias diferentes tanto para identificar o P300 (considerando a morfologia ${ }^{(2,4,7,9-10)}$, reprodutibilidade da onda ${ }^{(2,4,7,11-12)}$ e intervalo de latência $\left.{ }^{(2,4,7,13)}\right)$, como para marcar suas medidas de latência e amplitude esperadas para faixa etária avaliada (considerando 
o ponto de máxima amplitude da onda ${ }^{(3,9-10)}$, média entre duas replicações ${ }^{(3,14)}$, ponto mais próximo a $300 \mathrm{~ms}^{(5)}$, ponto da onda mais replicável $\left.{ }^{(3,10-12)}\right)$.

A latência deve ser medida como o tempo que decorre desde a aplicação do estímulo até o instante em que a componente correspondente ao P300 atinge a amplitude máxima, significando a ativação neural de resposta ao estímulo, por isso a importância de mensurá-la $a^{(3)}$.

O potencial evocado relacionado a eventos é uma superposição de todas as correntes elétricas do cérebro, sendo difícil determinar os locais com maior contribuição a partir dos sinais eletroencefalográficos, pois diferentes geradores neurológicos contribuem para a formação do P300. Esses são ativados simultaneamente e cada um é responsável por processar um aspecto específico do estímulo ou informação ${ }^{(3,5,15-17)}$. A amplitude e a latência representariam o resultado dos efeitos relacionados a três independentes categorias de variáveis psicológicas: probabilidade subjetiva, característica do estímulo e total de informação transmitida. Isto explicaria os trabalhos relacionando os vários locais de origem do P300: hipocampo, formação reticular mesencefálica, tálamo medial, córtex pré-frontal e área de associação parieto-temporal ${ }^{(2,18)}$, representando uma forma de acesso à memória, na qual a ativação de um dado processador estaria na dependência da memória adquirida ${ }^{(6,16)}$.

O estudo do P300 torna-se útil no diagnóstico de disfunções mentais, nomeadamente demência ${ }^{(9)}$, doença de Alzheimer ${ }^{(19)}$, doença de Parkinson ${ }^{(20)}$, no estudo de desordens clínicas com consequências a nível cognitivo como o alcoolismo; a esquizofrenia; o autismo; a esclerose múltipla ${ }^{(21)} \mathrm{e}$ a desordem de processamento auditivo ${ }^{(3)}$, além de alterações de aprendizagem $^{(22)}$.

Dentre os autores que pesquisaram o P300, três se destacam por fornecerem valores de latência e amplitude padronizados. O primeiro pesquisou indivíduos adultos americanos, testados em aparelho com quatro canais de registro seguindo os parâmetros a serem observados e mensurados: a presença/ausência das diversas ondas; latência de $\mathrm{N}_{1}, \mathrm{P}_{2} \mathrm{e}$ P300; e amplitude de $\mathrm{N}_{1}-\mathrm{P}_{2}, \mathrm{P}_{2}-\mathrm{P} 300$ e $\mathrm{N}_{1}-\mathrm{P} 300$. De acordo com a morfologia, as ondas devem estar presentes e serem duplicáveis. Os parâmetros utilizados foram: polaridade positiva, filtro passa alto de $20 \mathrm{~Hz}$ e passa baixo de $0,5 \mathrm{~Hz}$, janela de $500 \mathrm{~ms}$ e ganho de $10^{4}$. A latência de $\mathrm{N}_{1}$ deve ser menor que $120 \mathrm{~ms}$, a latência de $\mathrm{P}_{2}$ deve ser menor que 228 ms e a latência de $\mathrm{P} 300$ deve encontrar-se entre $250 \mathrm{~ms}$ a 350 ms e a amplitude entre 10 e $20 \mathrm{uV}$. A amplitude entre $\mathrm{N}_{1}-\mathrm{P}_{2}$ deve ser menor que $50 \%$ para os eletrodos $\mathrm{C}_{3}-\mathrm{C}_{4}$ ou $\mathrm{C}_{5}-\mathrm{C}_{6}$, sendo $\mathrm{C}$ correspondente à região central ou vértex do córtex no sistema 10/20 (diferença inter-hemisférios) $)^{(4)}$.

$\mathrm{O}$ segundo pesquisador utilizou aparelho com três canais de registro, sendo que os eletrodos foram colocados em $\mathrm{A}_{1}(-)$, $\mathrm{A}_{2}(-), \mathrm{F}_{\mathrm{P}_{\mathrm{z}}}$ (terra), $\mathrm{F}_{\mathrm{z}}(+), \mathrm{C}_{\mathrm{z}}(+)$ e $\mathrm{P}_{\mathrm{z}}(+)$ Neste estudo foi utilizado protocolo com taxa de replicação de um estímulo por segundo, janela de $700 \mathrm{~ms}$, filtro Notch ligado, filtro passa alto de $50 \mathrm{~Hz}$ e passa baixo de $0,05 \mathrm{~Hz}$, ganho de $10^{4}$, estímulo tone burst com platô de $50 \mathrm{~ms}$ e tempo para alcançar este plateau de 25 ms, intensidade dos estímulos de 60 a 70 dBNS, sendo $80 \%$ de estímulos frequentes e $20 \%$ de estímulos infrequentes. Foram observados os valores de latência, amplitude e morfologia das ondas. Os valores de normalidade encontrados para latência do P300 foram entre 220 e 380 ms e para amplitude média de $12 \mathrm{uV}^{(2)}$.

O terceiro estudo utilizou aparelho com dois canais de registro e os eletrodos foram colocados em $\mathrm{M}_{1}(-), \mathrm{M}_{2}(-)$, $\mathrm{F}_{\mathrm{z}}(\mathrm{comum}), \mathrm{C}_{\mathrm{z}}(+)$ e $\mathrm{P}_{\mathrm{z}}(+)$. O protocolo utilizado apresentava polaridade alternada, rate de 1,1 estímulo por segundo, janela de $500 \mathrm{~ms}$, filtro Notch ligado, filtro passa alto de 30 a $100 \mathrm{Hze}$ passa baixo de 1 a $3 \mathrm{~Hz}$, ganho de $10^{5}$, estímulo tone burst com platô de $30 \mathrm{~ms}$ e rise-fall de $10 \mathrm{~ms}$, sendo o estímulo freqüente de $1000 \mathrm{~Hz}$ e o raro de $2000 \mathrm{~Hz}$, na intensidade de $70 \mathrm{dBNA}$ e $85 \%$ dos estímulos freqüentes e $15 \%$ dos estímulos raros. Foram observados valores de latência e amplitude e morfologia das ondas. Encontrou-se para valores de latência o intervalo de 225 a $365 \mathrm{~ms}$ e amplitude entre 5 a $20 \mathrm{uV}^{(7)}$.

Espera-se que os valores encontrados neste estudo sejam equivalentes aos valores encontrados pela literatura pesquisada, uma vez que a população estudada não possui alterações sistêmicas, neurológicas e auditivas e que os padrões de protocolo são equivalentes aos da literatura pesquisada.

Em um estudo realizado com 50 adultos jovens saudáveis foram encontrados valores máximos das latências $\mathrm{N}_{1}$ até 125,7 $\mathrm{ms}, \mathrm{P}_{2}$ até $182 \mathrm{~ms}, \mathrm{~N}_{2}$ até $286,7 \mathrm{~ms}$ e $\mathrm{P} 300$ até $352,2 \mathrm{~ms}^{(11)}$.

O P300 em adultos normais foi estudado em 25 indivíduos normais (sem alterações de enfermidades sistêmicas ou psiquiátricas) com idades variando de 22 a 58 anos, com eletrodos colocados nos pontos $\mathrm{F}_{\mathrm{z}}$ e $\mathrm{C}_{\mathrm{z}}$. Verificou-se que a média do registro da onda do $\mathrm{P} 300$ no eletrodo $\mathrm{F}_{\mathrm{z}}$ foi de $321,24 \mathrm{~ms}$ e a média de 315,08 ms no eletrodo $\mathrm{C}_{\mathrm{z}}^{(23)}$.

Embora seja considerado um método objetivo de avaliação, o P300 pode sofrer interferência de alguns fatores que contribuem para a variabilidade de suas medidas. Fatores ambientais e a forma de realização do exame são variáveis que podem interferir nas respostas do P300 tais como: a ingestão recente de comida pode diminuir a latência e aumentar a amplitude, a temperatura corporal alterada leva a um desvio na latência, e a amplitude é maior nas épocas do ano em que há mais luz ${ }^{(3,10)}$. Em mulheres a amplitude do P300 pode apresentar valores ligeiramente superiores devido ao tamanho do crânio e uma menor espessura do mesmo $^{(3,10)}$, além da concentração de estrogênio, nas diferentes fases do ciclo menstrual, também pode influenciar na latência do P300 ${ }^{(24)}$. A interação entre estímulo e latência de P300 foi evidenciada, em uma relação inversamente proporcional, aumentando a intensidade do estímulo diminui a latência ${ }^{(3,14,25)}$. De acordo com esse estudo é conveniente verificar a forma que o indivíduo realizou a tarefa, e executar a repetição do exame, pois, a principal dificuldade na identificação do P300 resulta do fato de cerca de 20 a $30 \%$ dos indivíduos apresentarem um pico bífido - um primeiro pico, P300a, normalmente de maior amplitude nos eletrodos frontais e centrais, e um segundo pico, P300b. O P300a está ligado a processos de alerta imediato, enquanto que o $\mathrm{P} 300 \mathrm{~b}$ está relacionado com processos de atualização da memória ${ }^{(3,10)}$.

Além das variáveis já conhecidas e, portanto, controladas pelos examinadores, chama-nos a atenção a "subjetividade" envolvida na identificação e marcação da onda P300. Isto ocorre pela falta de um ou mais critérios de análise bem definidos e padronizados ${ }^{(10)}$. 
Com o aumento da idade foi observado aumento da latência e diminuição da amplitude de $\mathrm{P} 300$ e $\mathrm{N}_{2}^{(3,26)}$. Em indivíduos normais, jovens, o sinal de P300 foi mais evidente em $\mathrm{P}_{z}$ e em idosos, a distribuição foi semelhante nos três pontos $\left(\mathrm{F}_{z}, \mathrm{C}_{z} \text { e } \mathrm{P}_{z}\right)^{(13,27)}$. Outro estudo realizado com idosos comprovou que não houve diferença significativa da latência do $\mathrm{P} 300$ nas derivações $\mathrm{C}_{\mathrm{z}}$ e $\mathrm{P}_{\mathrm{z}}$ em relação ao sexo masculino e feminino, em indivíduos com e sem queixa auditiva, com e sem alteração da audiometria tonal limiar, em indivíduos com processamento auditivo normal e alterado, com e sem doenças sistêmicas ${ }^{(6)}$.

O P300 foi realizado em 20 sujeitos (dez do sexo feminino e dez do sexo masculino) otologicamente normais, sem história neurológica, de aprendizagem e stress (no momento do exame). A pesquisa dos potenciais evocados de longa latência foi realizada por meio do Programa Ati-Nautilus PE (versão 4,19 c-LERMED S.RI - Argentina, 1992), no estudo dos potenciais cognitivos (P300). Os valores médios de latência e amplitude variaram consideravelmente na população avaliada; foram encontradas diferenças significantes nas latências $\left(\mathrm{N}_{1}, \mathrm{P}_{2}\right.$ e $\left.\mathrm{N}_{2}\right)$ e amplitude $\left(\mathrm{N}_{1}\right)$, entre os indivíduos de um e de outro sexo. Não foi encontrada diferença significante para $P_{3}(p=0,31)$, provavelmente em decorrência do tamanho da amostra. Isso indica que as latências dos componentes $\mathrm{N}_{1}, \mathrm{P}_{2}$ e $\mathrm{N}_{2}$ foram menores em indivíduos do sexo feminino em relação aos do sexo masculino ${ }^{(12)}$.

Com o intuito de comparar se as atividades que envolvem mais complexidade influenciam nos valores de latência e amplitude do P300 foram avaliados indivíduos jovens e adultos saudáveis através da proposta de outra resposta frente aos estímulos raros, ou seja, ao invés de contá-los oralmente, apenas levantar uma das mãos. Ao analisar os procedimentos de avaliação do P300 comparados as diferentes instruções frente à percepção dos sons raros, foram constatadas que os componentes $\mathrm{N}_{1}, \mathrm{P}_{2}, \mathrm{~N}_{2}$ não se modificaram, mas o $\mathrm{P} 300$ apresentou uma ligeira modificação, demonstrando ser mais precoce no procedimento de menos complexidade cognitiva, o que justifica a padronização para uma nova proposta de resposta $^{(28)}$.

Com o destaque nas pesquisas atuais, como um instrumento de investigação clínica do processamento auditivo central, relacionado principalmente com os processos cognitivos, e o crescimento da utilização do Potencial Evocado Auditivo de Longa Latência em Belo Horizonte foi necessário estabelecer um padrão normativo para realização do exame P300 no Aparelho AMPLAID MK22 pertencente ao Centro Clínico de Fonoaudiologia da PUC-Minas, o que será essencial para futuros estudos.

O objetivo geral desta pesquisa foi comparar os valores de amplitude e latência de P300, por aparelho de dois canais de registro, em adultos jovens brasileiros saudáveis, sem alterações auditivas e neurológicas, com os valores de literatura. Os objetivos específicos foram verificar se os valores encontrados na casuística possuem correlação com o sexo; situação de realização do exame, apenas contando os estímulos raros e contando os estímulos raros e levantando a mão; e fases do ciclo menstrual. Essas análises foram realizadas a fim de estabelecer um padrão normativo para futuros estudos.

\section{MÉTODOS}

\section{Sujeitos}

A amostra constou de 22 indivíduos saudáveis, dez homens e 12 mulheres, sem antecedente ou queixa de perda de memória, de desatenção, sem problemas neurológicos e auditivos, de 18 a 31 anos de idade que assinaram o Termo de Consentimento Livre e Esclarecido para normatização do P300 no Centro Clínico de Fonoaudiologia da Pontifícia Universidade Católica de Minas Gerais (PUC-Minas). Esta pesquisa está de acordo com o Comitê de Ética em Pesquisa da PUC-Minas (CEP PUC), sob o número de registro CAAE 0036.0.213.000-05.

Todos os indivíduos foram submetidos à avaliação audiológica básica a fim de excluir aqueles com perda de audição.

\section{Procedimento}

Primeiramente foi realizada anamnese audiológica com o objetivo de descartar patologias auditivas associadas já na entrevista inicial. Em seguida, os indivíduos foram submetidos à avaliação audiológica básica que consistiu de audiometria tonal, audiometria vocal e imitanciometria, protocolos de avaliação, segundo Davis e Silvermann ${ }^{(29)}$ e Jerger $^{(30)}$.

As Respostas Auditivas de Longa Latência (P300) foram realizadas com equipamento AMPLAID MK22, de dois canais e em ambiente silencioso e com pouca luz.

Após acomodarmos o paciente em uma maca e com os olhos fechados, foi realizada a limpeza da pele com pasta abrasiva Every Per La Pulizia Della Cute, sendo fixados eletrodos na região do vértex $\left(\mathrm{C}_{\mathrm{z}}\right)$, parietal $\left(\mathrm{P}_{\mathrm{z}}\right)$, terra $\left(\mathrm{F}_{\mathrm{Pz}}\right)$, nos lóbulos das orelhas esquerda $\left(\mathrm{A}_{1}\right)$ e direita $\left(\mathrm{A}_{2}\right)$ conectados por cabos (jumper - A+) conforme sistema 10/20 entre eles. Foi usada uma pasta eletrolítica Elektrodem Creme da $\mathrm{Ge}$ Medical Systems entre a pele e o eletrodo para melhor condutividade da corrente elétrica. Os eletrodos foram ligados ao pré-amplificador e foi verificada a impedância que se manteve inferior a $5 \mathrm{~K} \Omega$.

O tipo de estímulo utilizado foi o tone burst com apresentação binaural, platô de $20 \mathrm{~ms}$ e rise-fall de $10 \mathrm{~ms}$, utilizando a freqüência de $1000 \mathrm{~Hz}$ como estímulo freqüente e o de 2000 $\mathrm{Hz}$ o raro. A intensidade apresentada foi de $80 \mathrm{dBNA}$ e cada estímulo apresentou duração de $200 \mathrm{~ms}$, sendo o rise-decay de $50 \mathrm{~ms}$. Para cada exame foram apresentados 500 estímulos, sendo $80 \%$ de freqüentes e $20 \%$ de raros.

Foram utilizados os parâmetros: polaridade positiva, filtro passa alto de $20 \mathrm{~Hz}$ e passa baixo de $0,5 \mathrm{~Hz}$, janela de $500 \mathrm{~ms}$ com $50 \mathrm{~ms}$ iniciais (pré-análise), e ganho de $10^{4}$.

A taxa de apresentação foi de intervalos regulares de um segundo, e os indivíduos orientados a contar toda vez que o estímulo raro foi apresentado. O eletroencefalograma foi ampliado em 50.000 vezes.

Antes de iniciar o exame, foi realizado um treino em que os estímulos foram apresentados para os indivíduos se familiarizarem com o teste.

O teste foi realizado em duas situações, a primeira situação apenas contando os estímulos raros (situação 1), e a segunda 
situação, em seguida à primeira, contando e levantando uma das mãos (situação 2). Foram utilizadas duas situações a fim de verificarmos se ocorrem diferenças nos valores de latência e amplitude quando a forma de realização do exame é modificada.

Foram medidas a latência e amplitude do P300 e geralmente, o primeiro pico positivo após $220 \mathrm{~ms}$ foi medido, sendo facilmente identificado.

Todos os exames foram gravados imediatamente após suas realizações. A análise foi realizada em um segundo momento, após a realização de todos os 22 exames, num mesmo dia, a fim de evitar divergências nas interpretações clínicas dos exames.

Para análise dos dados, as ferramentas estatísticas utilizadas foram análises descritivas (média, desvio padrão, mediana e medidas de máximo e mínimo e gráfico de ações), teste t de Student independente e análise de variância (ANOVA) considerando a situação (apenas contando; e contando e levantando a mão) e o sexo como variáveis. O nível de significância adotado foi menor que 0,05 .

As estatísticas descritivas foram realizadas separadamente para cada situação, para a latência tanto como para amplitude, e quanto ao sexo. O gráfico de ações permite comparar os diferentes intervalos obtidos neste estudo com os intervalos obtidos na literatura padrão.

\section{RESULTADOS}

Os resultados obtidos estão apresentados em forma de tabela para facilitar a visualização (Tabela 1).
A Figura 1 expõe a comparação dos valores de latência encontrados na casuística com os valores de latência encontrados pela literatura pesquisada.

Os exames de P300 realizados nesta pesquisa foram relacionados com os intervalos de latência propostos pela literatura. Dentre os exames realizados na situação 1, 21 exames se enquadram no intervalo entre 220 e $380 \mathrm{~ms}^{(2)}, 18$ no intervalo entre 225 a $365 \mathrm{~ms}^{(7)}$ e 14 no intervalo entre 250 a $350 \mathrm{~ms}^{(4)}$. Dos exames realizados na situação 2,20 se enquadram no intervalo entre 220 e $380 \mathrm{~ms}^{(2)}, 19$ no intervalo entre 225 a $365 \mathrm{~ms}^{(7)} \mathrm{e} 13$ no intervalo entre 250 a $350 \mathrm{~ms}^{(4)}$.

A Figura 2 expõe a comparação dos valores de amplitude encontrados na casuísta com os valores de amplitude propostos pela literatura pesquisada, com exceção do segundo pesquisador $^{(2)}$, relatado na revisão de literatura, pois o mesmo analisa a amplitude através de médias e não por meio de intervalos.

Os exames de $\mathrm{P} 300$ realizados foram também relacionados com os valores de amplitude propostos pela literatura. Dos exames realizados na situação 1,17 se enquadram no intervalo entre 5 a $20 \mathrm{uV}^{(7)}$ e 10 no intervalo entre 10 e $20 \mathrm{uV}^{(4)}$. Na situação 2, há 16 exames que se enquadram no intervalo entre 5 a $20 \mathrm{uV}^{(7)}$ e 9 no intervalo entre 10 e $20 \mathrm{uV}^{(4)}$. A média de amplitude dos exames da nossa casuística foi relacionada ao valor de média $12 \mathrm{uV}^{(2)}$, sendo na situação 1 a média 10,48 uV, e na situação 2 a média 9,09 uV.

A Tabela 2 analisa a variância para a latência e amplitude do P300, relacionando sexo e situação. Nesta é observado que o valor-p para a variável sexo em relação à medida de amplitude é p=0,049, sendo estatisticamente significativo.

Tabela 1. Medidas descritivas de latência e amplitude nas duas situações para homens e mulheres

\begin{tabular}{|c|c|c|c|c|c|c|c|c|c|}
\hline \multirow[t]{2}{*}{ Casuística } & \multirow[t]{2}{*}{ Situações } & \multicolumn{4}{|c|}{ Latência (ms) } & \multicolumn{4}{|c|}{ Amplitude (uV) } \\
\hline & & Média & DP & Mediana & Intervalo & Média & $\mathrm{DP}$ & Mediana & Intervalo \\
\hline \multirow[t]{2}{*}{ Homens e mulheres } & $A C$ & 337,55 & 28,32 & 340 & $256-384$ & 10,47 & 5,69 & 9,89 & $1,91-24,97$ \\
\hline & $\mathrm{CL}$ & 341,18 & 33,03 & 341 & $270-412$ & 9,09 & 5,20 & 8,43 & $1,24-18,49$ \\
\hline \multirow[t]{2}{*}{ Homens } & $A C$ & 332,40 & 20,63 & 331 & $294-366$ & 8,90 & 5,21 & 8,08 & $1,91-17,14$ \\
\hline & $\mathrm{CL}$ & 334,8 & 32,8 & 333 & $270-388$ & 7,10 & 5,81 & 5,43 & $1,24-18,49$ \\
\hline \multirow[t]{2}{*}{ Mulheres } & $\mathrm{AC}$ & 341,83 & 33,75 & 348 & $256-384$ & 11,79 & 5,95 & 11,36 & $3,24-24,97$ \\
\hline & $\mathrm{CL}$ & 346,50 & 33,75 & 346 & $278-412$ & 10,75 & 4,16 & 12,33 & $3,40-16,78$ \\
\hline
\end{tabular}

Legenda: $\mathrm{AC}=$ apenas contando; $\mathrm{CL}=$ contando e levantando a mão; $\mathrm{DP}=$ desvio padrão

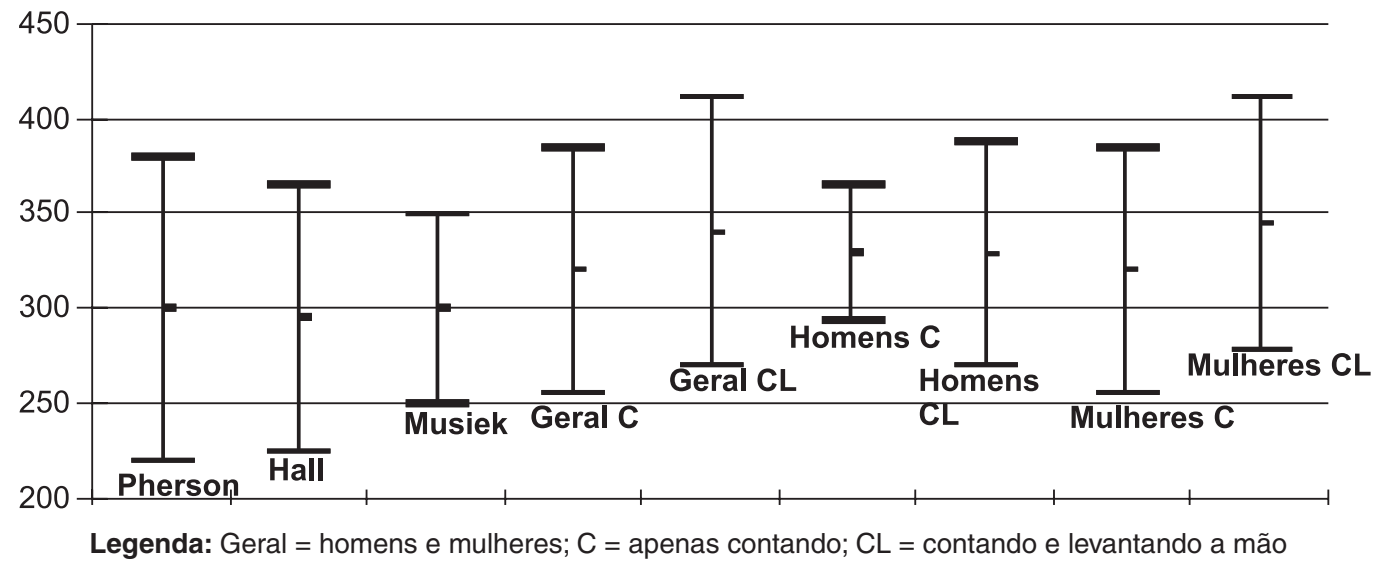

Figura 1. Comparação dos valores de latência encontrados na amostra com os valores da literatura 
A análise de variância para latência e amplitude relacionando o ciclo menstrual e a situação está representada na Tabela 3. Nesta o valor-p para a variável ciclo menstrual em relação à medida de amplitude é $\mathrm{p}=0,016$, sendo estatisticamente significativo.
Os valores médios de amplitude nas duas situações obtidos pelos homens e mulheres foram: para os homens 7,998 uV e para as mulheres $11,272 \mathrm{uV}$.

Foram pesquisados os valores médios de amplitude em cada fase do ciclo menstrual. No início do ciclo a média foi

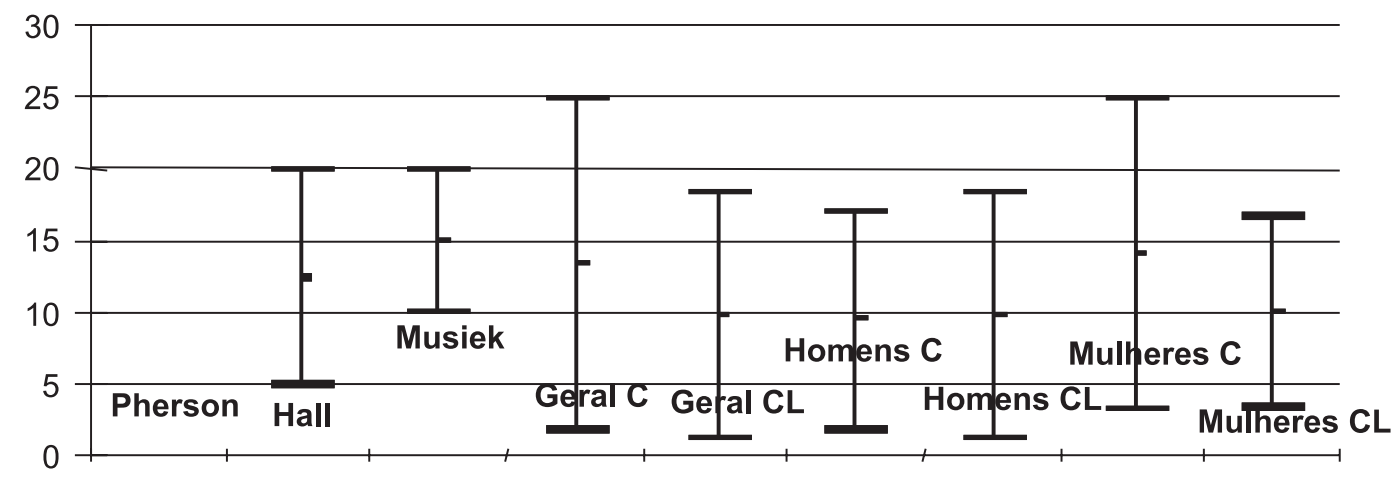

Legenda: Geral = homens e mulheres; $\mathrm{C}=$ apenas contando; $\mathrm{CL}=$ contando e levantando a mão

Figura 2. Comparação dos valores de amplitude encontrados na amostra com os valores da literatura

Tabela 2. Análise de variância para latência e amplitude, relacionando o sexo e a situação

\begin{tabular}{|c|c|c|c|c|c|c|c|}
\hline & Variáveis & DF & Seq SS & Adj SS & Adj MS & $\mathrm{F}$ & Valor de $p$ \\
\hline \multirow[t]{5}{*}{ Latência } & Si & 1 & 145,5 & 136,2 & 136,2 & 0,14 & 0,709 \\
\hline & Sx & 1 & 1218,0 & 1218,0 & 1218,0 & 1,26 & 0,268 \\
\hline & SiSx & 1 & 14,0 & 14,0 & 14,0 & 0,01 & 0,905 \\
\hline & $E$ & 40 & 38532,7 & 38532,7 & 963,3 & & \\
\hline & $\mathrm{T}$ & 43 & 39910,2 & & & & \\
\hline \multirow[t]{5}{*}{ Amplitude } & $\mathrm{Si}$ & 1 & 21,25 & 22,13 & 22,13 & 0,78 & 0,381 \\
\hline & Sx & 1 & 116,90 & 116,90 & 116,90 & 4,14 & $0,049\left(^{*}\right)$ \\
\hline & SiSx & 1 & 1,57 & 1,57 & 1,57 & 0,06 & 0,815 \\
\hline & $E$ & 40 & 1129,10 & 1129,10 & 28,23 & & \\
\hline & $\mathrm{T}$ & 43 & 1268,82 & & & & \\
\hline
\end{tabular}

ANOVA: $p<0,05$

Legenda: $\mathrm{Si}$ = situação; $\mathrm{Sx}=$ sexo; SiSx = situação X sexo; $\mathrm{E}=$ erro; $\mathrm{T}$ = total; $\mathrm{DF}$ (degrees of freedom) = grau de liberdade; Seq SS (sequential sums of squares) = soma dos quadrados em sequência; Adj SS (adjusted sums of squares) = soma dos quadrados ajustado; Adj MS (ajusted mean squares) = quadrado médio ajustado; $\mathrm{F}=$ estatística de teste; $\left({ }^{*}\right)$ valor de $\mathrm{p}$ estatisticamente significativo

Tabela 3. Análise de variância para latência e amplitude, relacionando o ciclo menstrual e a situação

\begin{tabular}{|c|c|c|c|c|c|c|c|}
\hline & Variáveis & DF & Seq SS & Adj SS & Adj MS & $\mathrm{F}$ & Valor de $p$ \\
\hline \multirow[t]{5}{*}{ Latência } & $\mathrm{Si}$ & 1 & 131 & 161 & 161 & 0,14 & 0,716 \\
\hline & $\mathrm{Ci}$ & 3 & 5638 & 5638 & 1879 & 1,60 & 0,229 \\
\hline & $\mathrm{SiCi}$ & 3 & 590 & 590 & 197 & 0,17 & 0,917 \\
\hline & $E$ & 16 & 18801 & 18801 & 1175 & & \\
\hline & $\mathrm{T}$ & 23 & 25159 & & & & \\
\hline \multirow[t]{5}{*}{ Amplitude } & $\mathrm{Si}$ & 1 & 6,56 & 34,20 & 34,20 & 2,06 & 0,171 \\
\hline & $\mathrm{Ci}$ & 3 & 231,17 & 231,17 & 77,06 & 4,63 & $0,016\left(^{*}\right)$ \\
\hline & $\mathrm{SiCi}$ & 3 & 83,16 & 83,16 & 27,72 & 1,67 & 0,214 \\
\hline & $E$ & 16 & 266,15 & 266,15 & 16,63 & & \\
\hline & $\mathrm{T}$ & 23 & 587,04 & & & & \\
\hline
\end{tabular}

Legenda: $\mathrm{Si}$ = situação; $\mathrm{Ci}$ = ciclo; $\mathrm{SiCi}$ = situação $\mathrm{X}$ ciclo; $\mathrm{E}=$ erro; $\mathrm{T}$ = total; $\mathrm{DF}$ (degrees of freedom) = grau de liberdade; Seq SS (sequential sums of squares) = soma dos quadrados em sequência; Adj SS (adjusted sums of squares) = soma dos quadrados ajustado; Adj MS (ajusted mean squares) = quadrado médio ajustado; $\mathrm{F}=$ estatística de teste; $\left(^{*}\right)$ valor de $\mathrm{p}$ estatisticamente significativo 
$8,683 \mathrm{uV}$, no meio do ciclo 4,554 uV, no final do ciclo 3,554 $\mathrm{uV}$ e na fase menstruada a média foi $3,553 \mathrm{uV}$.

\section{DISCUSSÃO}

A análise da variância para latência e amplitude relacionando as variáveis sexo e situação, evidenciou que a variável sexo não influenciou significativamente no valor médio de latência, concordando com dois estudos ${ }^{(6,12)}$ que não encontraram diferenças nos valores de latência de P300 entre os sexos. No entanto, com relação à amplitude, a variável sexo influenciou significativamente, estando o valor da amplitude média maior para os indivíduos femininos - 11,79 uV (Tabela 1). A interação entre as variáveis, situação e sexo, não influenciaram no valor médio de latência e amplitude. A variável situação não influenciou significativamente nos valores de latência e amplitude, demonstrando que não existem diferenças relevantes entre as situações 1 e 2 (Tabela 2), diferindo do encontrado na literatura ${ }^{(28)}$. Durante a realização dos exames os indivíduos pesquisados relataram achar mais fácil contar os estímulos quando os mesmos eram associados ao ato motor de levantar a mão, por isso acreditamos que essa metodologia pode ser adotada para indivíduos com dificuldades em realizar o exame apenas contando em sequiência.

Ao analisarmos a latência e a amplitude relacionadas ao ciclo menstrual, à situação e a associação entre eles, verificamos que somente a fase do ciclo menstrual influenciou os valores de amplitude (Tabela 3). No nosso estudo não foi evidenciado a influência do ciclo menstrual nos valores de latência, porém isso foi demonstrado por uma pesquisa ${ }^{(24)}$ que verificou a latência aumentada com a concentração de estrogênio nas fases do ciclo menstrual em mulheres saudáveis.

A metodologia adotada nesta pesquisa é embasada principalmente na proposta de Musiek $^{(4)}$, porém os outros autores utilizam metodologias parecidas, preservando dados como as frequiências dos estímulos fornecidos no exame. Acreditados que embora nossa população tenha sido de adultos até 31 anos, o que diminui a possibilidade de aumento de latência em função da idade, os valores de latência encontrados nessa pesquisa, valor mínimo de $256 \mathrm{~ms}$ e valor máximo de 412 ms (Tabela 1), estão situados um pouco acima dos intervalos propostos pela literatura pesquisada (Figura 1). Este aumento de latência pode ter ocorrido devido a grande variabilidade individual e a possível influência do cansaço na realização do exame na situação 2, cujo maior valor foi de $412 \mathrm{~ms}$.

Considerando os indivíduos de ambos os sexos nas duas situações; mulheres nas duas situações e homens na situação 2, os valores máximos de latência ultrapassaram os valores de latência situados no intervalo de 220 e $380 \mathrm{~ms}^{(2)}$, de 250 a $350 \mathrm{~ms}^{(4)}$, de 225 a $365 \mathrm{~ms}^{(7)}$, valor máximo do P300 até 352,2 $\mathrm{ms}^{(11)} \mathrm{e}$ valor de média do $\mathrm{P} 300$ no eletrodo $\mathrm{F}_{\mathrm{z}}$ de $321,24 \mathrm{~ms}$ e de 315,08 ms no eletrodo $\mathrm{C}_{\mathrm{z}}^{(23)}$. Para homens na situação 1, os valores de latência ultrapassaram os valores de latência situados no intervalo de 250 a $350 \mathrm{~ms}^{(4)}$, de 225 a $365 \mathrm{~ms}^{(7)}$, valor máximo do P300 até $352,2 \mathrm{~ms}^{(18)}$ e valor de média do P300 no eletrodo $F_{z}$ de $321,24 \mathrm{~ms}$ e de $315,08 \mathrm{~ms}$ no eletrodo $\mathrm{C}_{\mathrm{z}}{ }_{\mathrm{z}}^{(23)}$, no entanto, enquadrou-se no intervalo entre 220 e $380 \mathrm{~ms}^{(2)}$.

Verificamos que os pontos centrais dos valores de latência situados nos intervalos propostos pela literatura estão muito próximos de $300 \mathrm{~ms}$, enquanto que, para todos os valores de latência situados no intervalo obtido na amostra os pontos centrais estão sem torno de $375 \mathrm{~ms}$. Comparando com os valores de latência situados nos intervalos da literatura os valores obtidos na casuística se adaptam melhor ao intervalo entre 220 e $380 \mathrm{~ms}^{(2)}$.

Ao analisarmos a amplitude com relação aos indivíduos de ambos os sexos na situação 1 e as mulheres também na situação 1 , os valores mínimos de amplitude de cada grupo não alcançaram os valores mínimos encontrados entre 10 e 20 $\mathrm{uV}^{(4)}$ e entre 5 a $20 \mathrm{uV}^{(7)}$, sendo que os valores máximos de amplitude desses grupos ultrapassaram os valores máximos encontrados por esses autores. Para indivíduos de ambos os sexos na situação 2; os homens em ambas as situações e as mulheres na situação 2 , os valores mínimos de amplitude também não alcançaram os valores mínimos entre 10 e $20 \mathrm{uV}^{(4)} \mathrm{e}$ entre 5 a $20 \mathrm{uV}^{(7)}$, sendo que os valores máximos enquadraram-se nos valores encontrados por esses autores. Assim, os valores mínimos de amplitude obtidos na amostra encontraram-se abaixo do limite inferior dos intervalos entre 10 e $20 \mathrm{uV}^{(4)} \mathrm{e}$ entre 5 a $20 \mathrm{uV}^{(7)}$.

Analisando a média de amplitude dos indivíduos de ambos os sexos nas situações analisadas separadamente, comparada com a média encontrada por $\mathrm{McPherson}^{(2)}$, que não relata intervalo de valores de amplitude e sim uma média desses valores, observou-se que a média de $12 \mathrm{uV}^{(2)}$ foi significativamente diferente para a situação 2 .

Os valores de amplitude da casuística, mínimo de 1,24 uV e máximo de 24,97 (Tabela 1), apresentaram intervalo maior quando comparados com os valores de intervalo entre $10 \mathrm{e}$ $20 \mathrm{uV}^{(4)}$ e entre 5 a $20 \mathrm{uV}^{(7)}$ (Figura 2). Acreditamos que isso ocorreu devido também a grande variabilidade, tendo sofrido interferência das variáveis sexo e ciclo menstrual.

\section{CONCLUSÃO}

Por meio da análise dos resultados deste estudo podemos concluir que:

1. O sexo, as situações, a fase do ciclo menstrual e as associações entre essas variáveis não interferiram nos valores de latência.

2. As situações e as associações entre as variáveis não influenciaram nos valores de amplitude, entretanto o sexo e a fase do ciclo menstrual interferiram significativamente nos valores da amplitude.

3. Os valores de latência da amostragem aproximaram-se aos valores de intervalos de latência entre 220 e 380 ms citados na literatura. Contudo, os intervalos para a amplitude obtidos na amostra não se enquadraram adequadamente aos valores de intervalo encontrados na literatura.

Para a realização de futuras pesquisas sugerimos que os valores de latência e amplitude de referência sejam os intervalos obtidos nas situações para os homens e mulheres em conjunto. Quando os indivíduos pesquisados forem capacitados a contar os estímulos raros em sequiência os valores utilizados devem ser os obtidos na situação 1 e caso os indivíduos tenham alguma dificuldade em realizar o exame nessa situação aconselhamos utilizar os valores obtidos na situação 2 . 


\section{AGRADECIMENTOS}

Agradecemos à neuropsicóloga Aila Reis, aos voluntários que participaram deste estudo, à coordenação do curso de Fonoaudiologia da PUC-Minas, e a todos que contribuíram para a realização desta pesquisa.

\begin{abstract}
Purpose: To compare the values of latency and amplitude of P300 of healthy Brazilian young adults with the data available on the literature, in order to establish a normative standard for future studies. In addition, the aim was also to verify if the values found in the sample were correlated to gender, situation proposed in the methodology of the examination, and phase of menstrual cycle. Methods: The sample consisted of 22 healthy individuals, without any attention complaints, neurological or auditory disorders, and with ages ranging from 18 to 30 years. All subjects carried out basic audiological evaluation in order to exclude those with hearing loss. The P300 test was carried out in a quiet room, using the two channels equipment AMPLAID MK22. Results: The latency values obtained in the sample, after compared with the literature reference, were in accordance with the latency intervals between 220 e $380 \mathrm{~ms}$. Only amplitude values were influenced by gender and phase of the menstrual cycle. Conclusion: The latency values of the sample were not influenced by the variables gender, methodology of the testing situation and menstrual cycle, and were in accordance to the latency interval values between 220 and $380 \mathrm{~ms}$. Amplitude values were influenced by gender and menstrual cycle, but were not in accordance to the values found in literature.
\end{abstract}

Keywords: Hearing tests; Evoked potentials, auditory; Event-related potentials, P300; Adult

\section{REFERÊNCIAS}

1. Bess FH, Humes LE. Fundamentos de audiologia. [Tradução de Marcos Domingues]. 2a ed. Porto Alegre: Artmed Editora; 1998.

2. McPherson DL. Late potentials of the auditory system. San Diego: Singular Publishing Group; c1996.

3. Silva A, Batista J, Carvalho V. Potenciais evocados [dissertação]. Lisboa: Departamento de Física da Faculdade de Ciências da Universidade de Lisboa; 1998.

4. Musiek FE, Baran JA, Pinheiro ML. Behavioral and electrophysiological test procedures. In: Musiek FE, Baran JA, Pinheiro ML. Neuroaudiology: case studies. San Diego: Singular Publishing Group; 1994. cap. 2. p. 7-28.

5. Halgren E, Squires NK, Wilson CL, Rohrbaugh JW, Babb TL, Crandall PH. Endogenous potentials generated in the human hippocampal formation and amygdala by infrequent events. Science. 1980;210(4471):803-5.

6. Nunes FB. Da avaliação do P300 e do processamento auditivo em pacientes idosos com e sem queixa auditiva [tese]. São Paulo: Universidade Federal de São Paulo. Escola Paulista de Medicina; 2002.

7. Hall JW. Handbook of auditory evoked responses. Boston: Allyn and Bacon; c1992.

8. Karniski W, Blair RC. Topographical and temporal stability of the P300. Electroencephalogr Clin Neurophysiol. 1989;72(5):373-83.

9. Polich J. Task difficulty, probability, and inter-stimulus interval as determinants of P300 from auditory stimuli. Electroencephalogr Clin Neurophysiol. 1987;68(4):311-20.

10. Junqueira CAO, Colafêmina JF. Investigação da estabilidade inter e intra-examinador na identificação do P300 auditivo: análise de erros. Rev Bras Otorrinolaringol. 2002;68(4):468-78.

11. César CPHAR, Munhoz MSL. Avaliação dos potenciais de longa latência relacionados a eventos em indivíduos jovens e adultos saudáveis. Acta AWHO. 1997;16(3):114-22.

12. Colafêmina JF, Fellipe ACN, Junqueira CAO, Frizzo AC. Potenciais evocados auditivos de longa latência (P300) em adultos jovens saudáveis: um estudo normativo. Rev Bras Otorrinolaringol. 2000;66(2):144-8.

13. Pfefferbaum A, Ford JM, Roth WT, Kopell BS. Age differences in P3-reaction time associations. Electroencephalogr Clin Neurophysiol. 1980;49(3-4):257-65.

14. Covington JW, Polich J. P300, stimulus intensity, and modality.
Electroencephalogr Clin Neurophysiol. 1996;100(6):579-84.

15. Johnson R Jr. On the neural generators of the P300 component of the event-related potential. Psychophysiology. 1993;30(1):90-7.

16. Smith ME, Halgren E, Sokolik M, Baudena P, Musolino A, LiegeoisChauvel C, Chauvel P. The intracranial topography of the P3 eventrelated potential elicited during auditory oddball. Electroencephalogr Clin Neurophysiol. 1990;76(3):235-48.

17. Munhoz MSL, Silva MLG, Ganança MM, Caovila HH, Frazza MM. Respostas auditivas de longa latência. In: Munhoz MSL, Caovilla HH, Silva MLG, Ganança MM, editores. Audiologia clínica. São Paulo: Atheneu; 2000. p.284. [Série otoneurológica, v.2].

18. Vaughan HG Jr, Ritter W. The sources of auditory evoked responses recorded from the human scalp. Electroencephalogr Clin Neurophysiol. 1970;28(4):360-7.

19. Williams PA, Jones GH, Briscoe M, Thomas R, Cronin P. P300 and reaction-time measures in senile dementia of the Alzheimer type. Br J Psychiatry. 1991;159:410-4.

20. Pineroli JCA, Campos DS, Wiemes GR, Meneses MS, Mocellin M. Avaliação auditiva central com BERA e P300 na Doença de Parkinson. Rev Bras Otorrinolaringol. 2002;68(4):462-6.

21. Casanova-González MF, Cabrera-Gómez JA, Aquino-Cías J, AneirosRivas R, Fernández-Bermúdez R. Evaluación neurofisiológica en pacientes con esclerosis múltiple clínica definida con especial referencia al estudio de la onda P300. Rev Neurol. 1999;29(12):1134-7.

22. Farias LS, Toniolo IF, Cóser PL. P300: avaliação eletrofisiológica da audição em crianças sem e com repetência escolar. Rev Bras Otorrinolaringol. 2004;70(2):194-9.

23. Franco GM. O potencial evocado cognitivo em adultos normais. Arq Neuropsiquiatr. 2001;59(2A):198-200.

24. Fukuda Y. P300 e ciclo menstrual em mulheres jovens normais [tese]. São Paulo: Universidade Federal de São Paulo. Escola Paulista de Medicina;1993.

25. Ritter W, Vaughan HG Jr, Costa LD. Orienting and habituation to auditory stimuli: a study of short term changes in average evoked responses. Electroencephalogr Clin Neurophysiol. 1968;25(6):550-6.

26. Goodin DS, Squires KC, Henderson BH, Starr A. Age-related variations in evoked potentials to auditory stimuli in normal human subjects. Electroencephalogr Clin Neurophysiol. 1978;44(4):447-58. 
27. Pfefferbaum A, Ford JM, Roth WT, Kopell BS. Age-related changes in auditory event-related potentials. Electroencephalogr Clin Neurophysiol. 1980;49(3-4):266-76.

28. César CPHAR, Munhoz MSL. O P300 auditivo em jovens e adultos saudáveis como uma nova proposta de resposta: levantar a mão. Acta AWHO. 1999;18(1):32-7.
29. Davis H, Silverman SR. Hearing and deafness. 3rd ed. New York: Holt, Rinehart \& Winston; 1970.

30. Jerger J. Clinical experience with impedance audiometry. Arch Otolaryngol. 1970;92(4):311-24. 